\title{
ANÁLISIS DEL CONSUMO DE SUSTANCIAS E INDICADORES DE SALUD FÍSICA Y PSICOLÓGICA EN HOMBRES Y MUJERES JOVENES
}

\author{
López-Fando-Galdón, María \\ Salusex. Departamento de Psicología Básica, Clínica y Psicobiología \\ Universitat Jaume I, Castellón \\ al320113@uji.es \\ Ballester-Arnal, Rafael \\ Salusex. Departamento de Psicología Básica, Clínica y Psicobiología \\ Universitat Jaume I, Castellón \\ García-Barba, Marta \\ Salusex. Departamento de Psicología Básica, Clínica y Psicobiología \\ Universitat Jaume I, Castellón \\ Elipe-Miravet, Marcel \\ Salusex. Departamento de Psicología Básica, Clínica y Psicobiología \\ Universitat Jaume I, Castellón \\ Bisquert-Bover, Mar \\ Salusex. Departamento de Psicología Básica, Clínica y Psicobiología \\ Universitat Jaume I, Castellón
}

RRecepción Artículo: 14 Febrero 2020

Admisión Evaluación: 4 marzo 2020

Informe Evaluador 1: 18 marzo 2020

Informe Evaluador 2: 20 Marzo 2020

Aprobación Publicación: 20 abril 2020

\section{RESUMEN}

La salud es una experiencia biopsicosocial que se relaciona con ciertos aspectos como los estilos de vida y el bienestar. Sin embargo, esta perspectiva no siempre se ha estudiado atendiendo tanto al género como a indicadores de salud física y mental. Objetivo: analizar la relación entre hábitos de salud específicos (tabaco, alcohol y consumo de sustancias) y la percepción subjetiva de bienestar físico y mental, teniendo en cuenta las diferencias de género. Método: 600 universitarios (50\% hombres; 50\% mujeres) completaron un cuestionario online sobre Estilos de Vida y Salud (Giménez-García \& Ballester-Arnal, 2017). Resultados: el 13,5\% reporta una mala salud física (14\% hombres y $13 \%$ mujeres) y el $17,7 \%$ una mala salud mental ( $18,3 \%$ hombres y $17 \%$ mujeres). En cuanto al consumo de sustancias tóxicas, el 14,7\% de la muestra fuma, el $47 \%$ se ha emborrachado bastantes-muchas veces y el $34,2 \%$ ha consumido otras sustancias. Más hombres que mujeres se han emborrachado muchas veces $\left(X^{2}=10,13 ; p=.017\right)$ y han consumido otras sustancias alguna vez $\left(X^{2}=8,08 ; p=, 004\right)$. En general, no existe una asociación entre el consumo y abuso de tabaco, alcohol y otras sustancias con la percepción de salud mental ni física por parte de los jóvenes. Así, aunque en teoría debería existir cierta relación entre estilos de vida como el consumo de sustancias y la salud mental y física, gran parte de estos resultados no han sido significativos. Dado que se ha evaluado percepción subjetiva de salud mental y física y no indicadores objetivos, 


\section{ANÁLISIS DEL CONSUMO DE SUSTANCIAS E INDICADORES DE SALUD FÍSICA Y PSICOLÓGICA EN HOMBRES Y MUJERES JÓVENES}

esto puede deberse a la baja percepción de riesgo de los jóvenes, lo que les lleva a la realización de conductas no saludables sin por ello ser conscientes de las consecuencias no fácilmente reconocibles a corto plazo. En los programas de prevención es fundamental considerar esta falta de percepción de riesgo entre los jóvenes.

Palabras clave: consumo de sustancias; estilos de vida; salud física; salud mental; género

\section{ABSTRACT}

Analysis of substance consumption and indicators of physical and psychological health in young men and women. Health is a biopsychosocial experience related to certain aspects such as lifestyles and well-being. However, this perspective has not always been studied with regard to both gender and physical and mental health indicators. Objective: to analyze the relationship between specific health habits (tobacco, alcohol and substance consumption) and the subjective perception of physical and mental well-being taking into account gender differences. Method: 600 university students (50\% men; 50\% women) completed an online questionnaire regarding Lifestyles and Health (Giménez-García \& Ballester-Arnal, 2017). Results: 13.5\% reported poor physical health (14\% men and $13 \%$ women) and $17.7 \%$ poor mental health (18.3\% men and $17 \%$ women). Regarding the consumption of toxic substances, $14.7 \%$ of the sample smokes, $47 \%$ of the sample has been quitemany times drunk and $34.2 \%$ of the sample has consumed other substances. More men than women have been drunk many times $\left(X^{2}=10.13 ; p=.017\right)$ and more men than women have ever used other substances $\left(X^{2}=8.08\right.$; $\mathrm{p}=.004$ ). In general, there is no association between the consumption and abuse of tobacco, alcohol and other substances with the perception of mental or physical health by young people. In spite of there should be a certain relationship between lifestyles such as substance use and mental and physical health, many of these results have not been significant. Given that subjective perception of mental and physical health has been evaluated and objective indicators have not, this may be a resault of the low risk perception of young people, which leads them to carry out unhealthy behaviors without being aware of the consequences at the short term. In prevention programs it is essential to consider this lack of risk perception at young people.

Keywords: drugs; lifestyle; physical health; mental health; gender

\section{INTRODUCCIÓN}

El consumo de sustancias legales e ilegales está ampliamente extendido en nuestra sociedad. Según los últimos datos aportados por el Observatorio Español sobre Drogas (2019), el mayor consumo sigue siendo de alcohol. Entre la población española, el $75.2 \%$ ha consumido alcohol en los últimos 12 meses y el 18.6\% se ha emborrachado en el último año, el $40.9 \%$ ha consumido tabaco y el 1.1\% ha consumido otras sustancias. Las prevalencias en el consumo de alcohol, tabaco, cannabis -sustancia ilegal cuyo consumo está más extendido entre los jóvenes- y cocaína son mayores en el grupo de 15 a 34 años. Así, el mayor inicio de consumo de otras drogas (cannabis y cocaína) tiene lugar entre los hombres menores de 25 años.

Más allá de las altas prevalencias, estas conductas de riesgo tienen un gran impacto para la salud (GiménezGarcía, Ruiz-Palomino, Gil-Llario, Ballester-Arnal y Castro, 2016). En concreto, el consumo de estas sustancias, conlleva el aumento del riesgo de diversas enfermedades (Lugones, Ramírez, Pichs y Miyar, 2006; Guzmán y Ramírez, 2006) y problemas cognitivos como la alteración de la atención, la memoria, la emoción y la toma de decisiones (Torres y Fiestas, 2012). De manera particular, fumar tiene efectos a corto plazo sobre algunos aspectos tan relevantes como la función pulmonar o la disminución de la aptitud física (U.S. Department of Health and Human Services, 2004). Si tenemos en cuenta otras drogas como el alcohol, se ha mostrado su repercusión en una serie de dolencias físicas, daño cerebral y déficits neurocognitivos, lo que implicaría el deterioro de procesos de aprendizaje, así como problemas emocionales (Brown et al., 2008). Además, otros estudios destacaron una asociación entre el consumo de sustancias psicoactivas y la presencia de trastornos mentales en la adolescencia (Cruz-Ramírez, Gómez-Restrepo y Rincón, 2018).

Por lo que respecta al género, los hombres inician antes tanto el consumo de alcohol, como las primeras borracheras, entre los 10 y 12 años (Giménez-García, Ruiz-Palomino, Gil-Juliá, Nebot-García y Ballester-Arnal, 
2018). Además, tanto los hombres como las mujeres que empezaron el consumo antes de los 14 años, lo hacen con más frecuencia y reportan en mayor grado haber sufrido, en algún momento, consecuencias negativas derivadas del alcohol. En otro estudio (Hernando, Oliva y Pertegal, 2013), se observó un aumento de consumo de alcohol en hombres y mujeres a medida que aumentaba la edad. Además, mientras las mujeres consumían más tabaco, los hombres consumían más cannabis, con las consecuencias físicas y psicosociales que ambos consumos pueden acarrear.

La literatura existente confirma la relación entre el consumo de sustancias y la salud general. Sin embargo, falta más evidencia sobre la posible existencia de diferencias de género en dicho impacto, así como sobre la propia percepción que las personas tienen de ese impacto en su salud física y mental. De hecho, algunos estudios muestran como el factor género algunas veces ha sido desatendido, aun mostrándose muy relevante en los esfuerzos preventivos (Östlin, 2005; Salmerón, Giménez y Nieto, 2016). Por tanto, el objetivo del presente estudio es analizar la relación entre hábitos de salud específicos (tabaco, alcohol y consumo de sustancias tóxicas) y la percepción subjetiva de bienestar físico y mental, desde una perspectiva de género.

\section{MÉTODO}

\section{Participantes}

Un total de 600 estudiantes universitarios participaron en el estudio, de los cuales el 50\% eran hombres y el $50 \%$ eran mujeres.

\section{Instrumento}

Para la recogida de los datos se administró un cuestionario sobre Estilos de vida y Salud (Giménez-García y Ballester-Arnal, 2017). Este cuestionario evalúa variables asociadas a la realización de conductas de salud como la percepción de riesgo, la intención de conducta, las motivaciones para realizar conductas saludables y la realización de las propias conductas. En concreto, se evalúan ámbitos como la alimentación y el ejercicio físico, el consumo de sustancias, la imagen corporal, la sexualidad, el uso de internet y móvil, los hábitos de sueño y la seguridad vial. Además, también se evalúa la estimación sobre la salud física, la salud mental y la calidad de vida en general. En este estudio se analizaron dos ítems de escala Likert relacionados con la percepción del estado de salud (estado salud física y mental), así como tres ítems que medían factores conductuales, dos de tipo dicotómico (consumo de tabaco y consumo de sustancias) y uno de escala Likert (frecuencia del consumo abusivo de alcohol). Este ítem ha sido seleccionado por ser más discriminativo que el consumo de alcohol.

\section{Procedimiento}

El estudio pertenece a uno más amplio cuyo objetivo era conocer y analizar los hábitos de salud en la población joven. Una vez obtenida la autorización para realizarlo, por parte de la Comisión Deontológica de la Universitat Jaume I, la información sobre el estudio fue difundida a través de redes sociales, con la finalidad de dar a conocerlo con la mayor cobertura posible. El mensaje incluía información relativa a las características y la finalidad del estudio. Seguidamente, se encontraba un enlace a la plataforma que permitía el acceso el cuestionario. Una vez habían accedido, procedían a dar el consentimiento informado aceptando su participación en el estudio de forma anónima y voluntaria y, finalmente, procedían a la cumplimentación del cuestionario.

\section{Análisis estadísticos}

Los datos se analizaron mediante el programa estadístico SPSS (versión 23). Se realizaron análisis descriptivos para caracterizar a la muestra. Posteriormente, se utilizaron contrastes Chi cuadrado para analizar las diferencias, entre hombres y mujeres, en la percepción de salud física y mental, además de en el consumo de alcohol, tabaco y otras sustancias. Estos análisis diferenciales, también se utilizaron para analizar la percepción de salud física y mental en función del consumo de tabaco, alcohol y otras sustancias, en hombres y mujeres. 


\section{ANÁLISIS DEL CONSUMO DE SUSTANCIAS E INDICADORES DE SALUD FÍSICA Y PSICOLÓGICA EN HOMBRES Y MUJERES JÓVENES}

\section{RESULTADOS}

\section{Consumo de sustancias según género}

Los análisis muestran que el 47\% de los participantes se ha emborrachado bastantes-muchas veces, siendo lo más frecuente haberse emborrachado alguna vez (Ver Figura 1). En cuanto a las diferencias entre hombres y mujeres, más hombres reportan haberse emborrachado muchas veces, mientras que más mujeres que hombres reportan no haberse emborrachado nunca, haberse emborrachado solo alguna vez y haberse emborrachado bastantes veces. Estas diferencias son significativas a nivel estadístico $\left(X^{2}=10.14 ; p=.017\right)$.

Figura 1. Distribución de la frecuencia de abuso del alcohol según género( $n=600)$

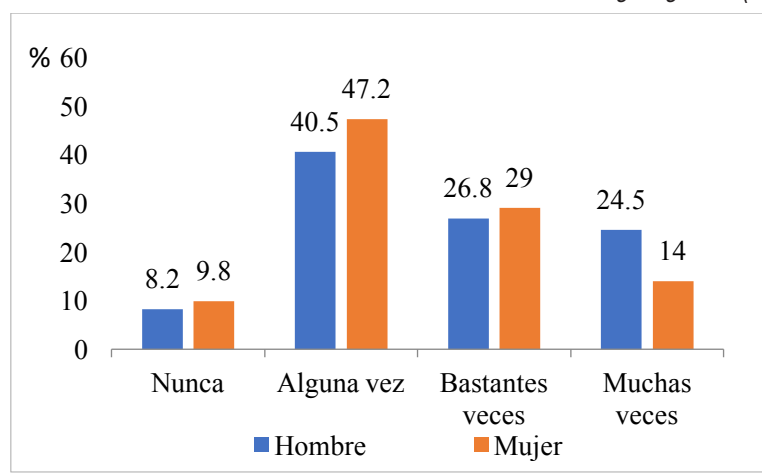

El 14,7\% de los participantes consume tabaco (Ver Figura 2), siendo el porcentaje de consumo de hombres y mujeres prácticamente idéntico, sin existir diferencias significativas a nivel estadístico $\left(X^{2}=0.53 ; p=.817\right)$. Por otro lado, al analizar el consumo de otras sustancias observamos que un $34,2 \%$ las ha consumido alguna vez, y que además, más hombres que mujeres han consumido sustancias en alguna ocasión, existiendo diferencias estadísticamente significativas $\left(X^{2}=8.08 ; p=.004\right)$.

Figura 2. Distribución de la frecuencia de consumo de tabaco y otras sustancias según género ( $n=600)$

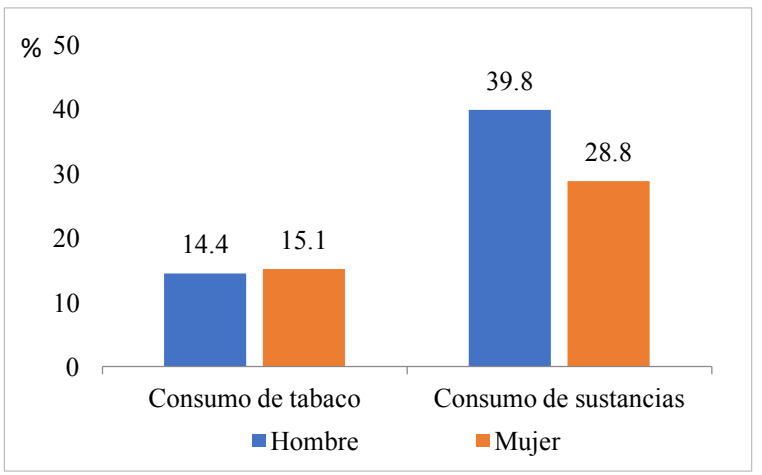

\section{Percepción de salud mental y física según género}

La mayoría de universitarios perciben una buena o muy buena salud mental, mientras que un $18.3 \%$ de hombres y un $17 \%$ de mujeres reporta tener una salud mental regular, mala o muy mala (ver Figura 3). Si tenemos en cuenta el género observamos que más mujeres perciben "buena salud mental" mientras que más hombres 
reportan "muy buena salud mental", siendo estas diferencias en general entre géneros no significativas $\left(X^{2}=\right.$ 3.86; $p=.426)$.

Figura 3. Distribución de la frecuencia de la percepción de salud mental según género ( $N=600)$.

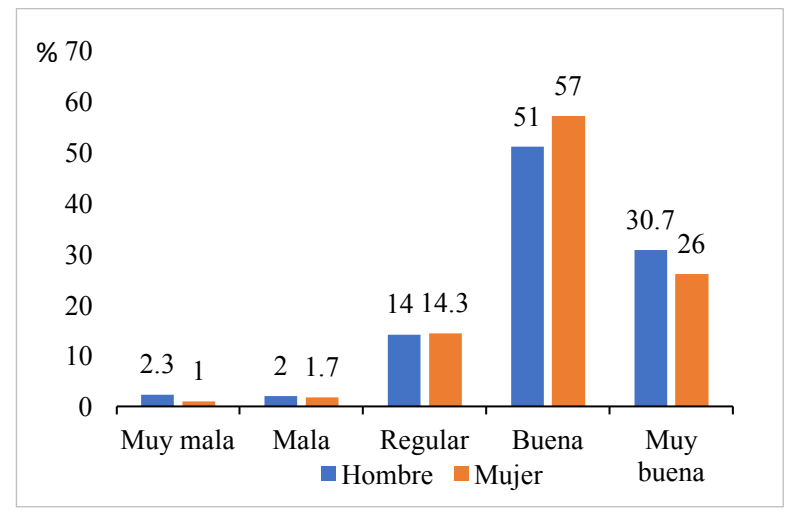

Al evaluar la percepción de salud física observamos que la mayoría de la muestra percibe tener una "buena o muy buena" salud física, mientras que un $13 \%$ de mujeres y un $14 \%$ de hombres reporta una salud física como "regular, mala o muy mala" (ver Figura 4). Si observamos las diferencias según el género, más mujeres perciben una buena salud física y superan algo a los hombres en la valoración como mala. Mientras tanto, más hombres que mujeres reportan una percepción muy buena del estado físico, así como regular y muy mala. Estas diferencias son significativas $\left(X^{2}=12.47 ; p=.014\right)$.

Figura 4. Distribución de la frecuencia de la percepción de salud física según género (n=600).

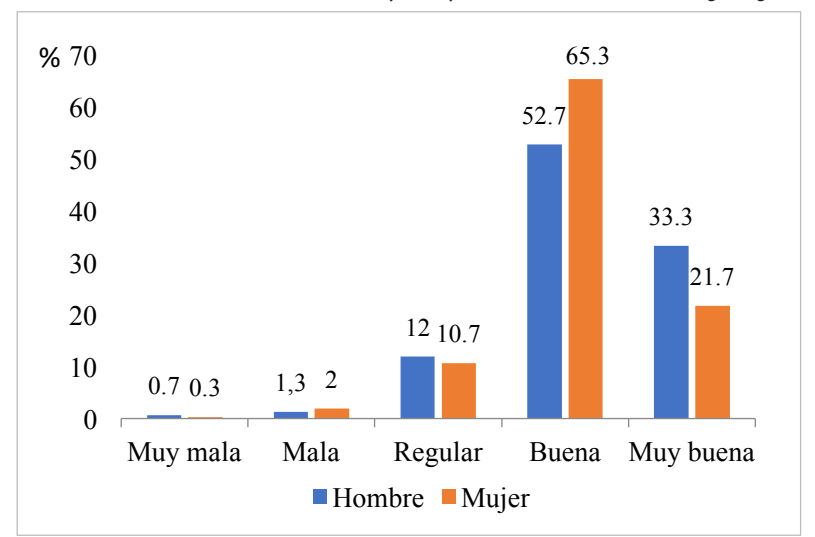

\section{Diferencias en la percepción de salud mental y física según consumo de alcohol, en mujeres y hombres}

Si analizamos la percepción de salud mental y el abuso de alcohol en mujeres (Ver Figura 5) vemos que la suma de los porcentajes de "buena y muy buena salud mental" es mayor en las que se han emborrachado algunas veces, seguida por las que lo han hecho bastantes veces, aunque no se han encontrado diferencias significativas $\left(X^{2}=16.32 ; p=.177\right)$. 


\section{ANÁLISIS DEL CONSUMO DE SUSTANCIAS E INDICADORES DE SALUD FÍSICA Y PSICOLÓGICA EN HOMBRES Y MUJERES JÓVENES}

Figura 5.

Distribución de la percepción de salud mental en mujeres, según la frecuencia de abuso del alcohol ( $n=300)$

$\% 80$

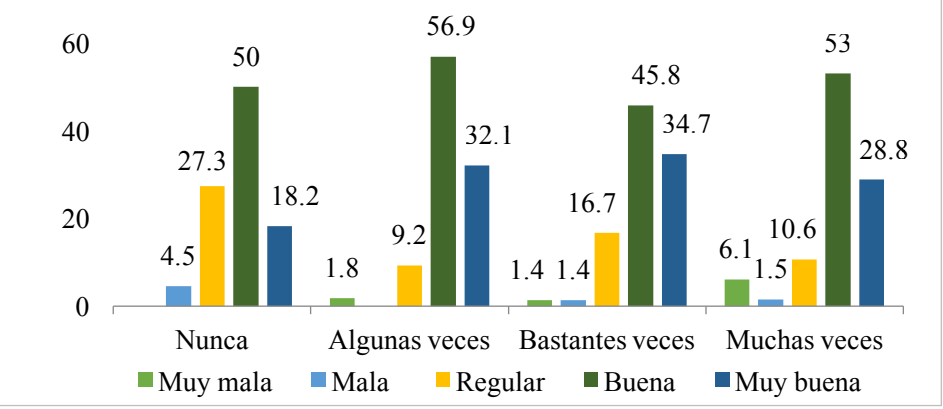

Al analizar la percepción de salud mental en hombres respecto al consumo de alcohol (Ver Figura 6), se aprecia que la suma de los que valoran su salud mental como buena y muy buena es mayor en los que se han emborrachado solo algunas veces, seguidos de los que no lo han hecho nunca, aunque de nuevo las diferencias, no alcanzaron significación estadística $\left(X^{2}=13.95 ; p=.304\right)$.

Figura 6 .

Distribución de la percepción de salud mental en hombres, según la frecuencia de abuso del alcohol (n=300)

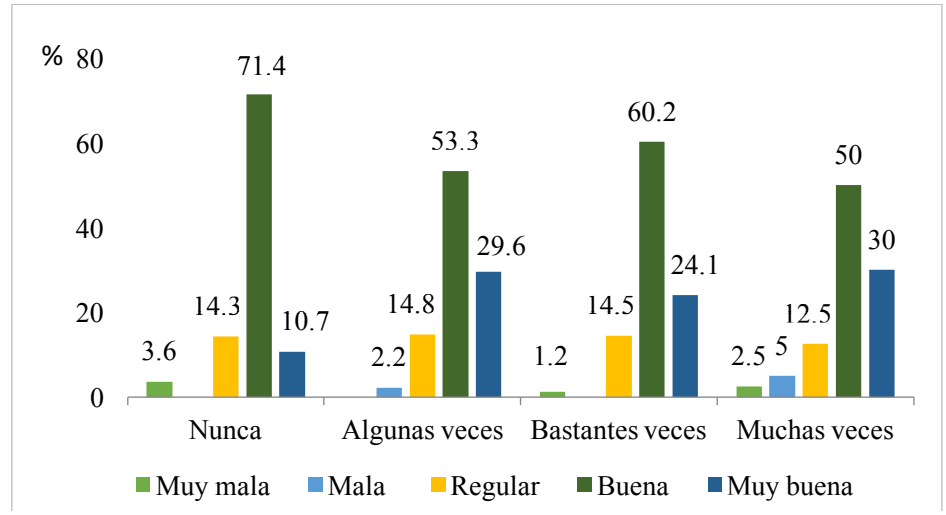

Además, se ha comprobado si existen diferencias en la percepción de salud física en mujeres y hombres en función de la frecuencia de veces que se ha abusado del alcohol. Se ha comprobado que la suma de las mujeres que valoran su salud física como buena y muy buena es mayor en las que se han emborrachado bastante veces, seguidas por las que lo han hecho muchas veces (Ver Figura 7). En cualquier caso, no se encuentran diferencias significativas $\left(X^{2}=9.63 ; p=.648\right)$. 
Figura 7.

Distribución de la percepción de salud física en mujeres, según la frecuencia de abuso del alcohol ( $n=300)$

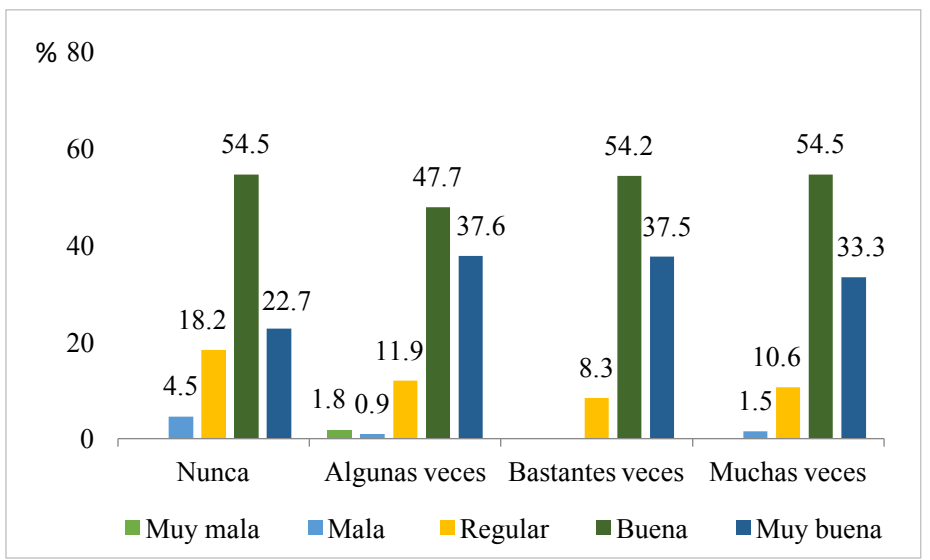

En hombres, sucede exactamente lo mismo (Ver Figura 8) no encontrándose tampoco diferencias significativas $\left(X^{2}=9.84 ; p=.629\right)$.

Figura 8.

Distribución de la percepción de salud física en hombres, según la frecuencia de abuso del alcohol ( $n=300)$

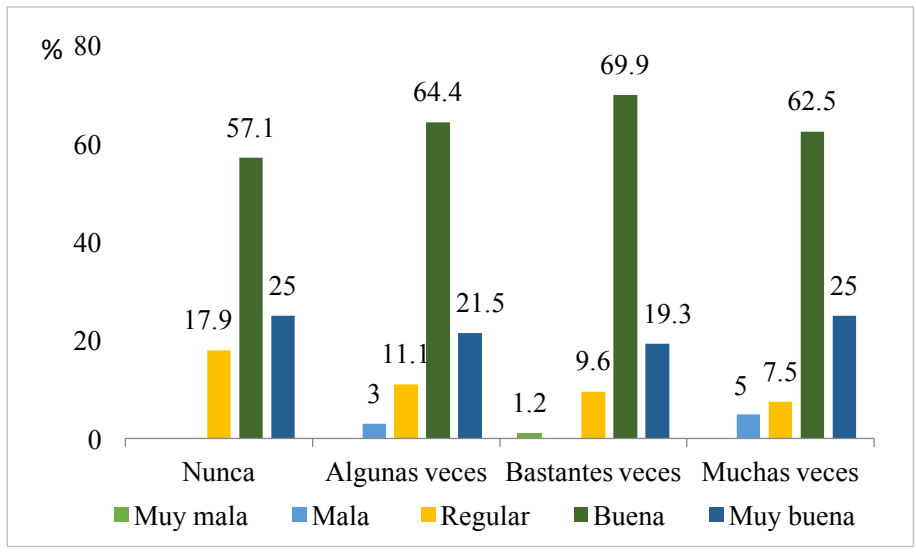

\section{Diferencias en percepción de salud mental y física según consumo de tabaco y otras sustancias, en mujeres y hombres}

Si analizamos la percepción de salud mental en mujeres que consumen tabaco y otras sustancias observamos que, las que consumen tabaco presentan una prevalencia más alta en percepción de buena salud mental ( $86.1 \%$ v. $80.9 \%$ ), respecto a las que no lo hacen, a diferencia de lo que sucede con el consumo de otras sustancias (78.1\% vs. 83.9\%) (Ver Figura 9). En cualquier caso, no existen diferencias significativas en la percepción de salud mental según el consumo de tabaco $\left(X^{2}=1.33 ; p=.855\right)$, ni de otras sustancias $\left(X^{2}=5.98 ; p=.201\right)$. 


\section{ANÁLISIS DEL CONSUMO DE SUSTANCIAS E INDICADORES DE SALUD FÍSICA Y PSICOLÓGICA EN HOMBRES Y MUJERES JÓVENES}

Figura 9 .

Distribución de percepción de salud mental en mujeres, según consumo de tabaco y otras sustancias $(n=300)$

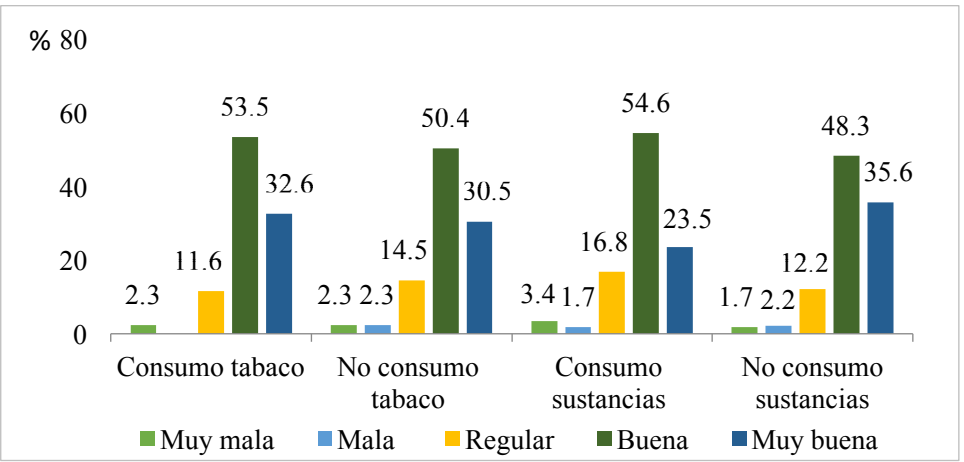

Respecto a la percepción de salud mental en los hombres (Ver Figura 10), observamos que aquellos que no consumen tabaco ni otras sustancias presentan una mayor prevalencia en la valoración de su salud mental como buena y muy buena ( $83.8 \%$ vs $77.8 \%$ en tabaco y $85.5 \%$ vs. $76.7 \%$ en otras sustancias), respecto a los que consumen. No obstante, las diferencias en la percepción mental según el consumo de tabaco $\left(X^{2}=7.24 ; p=.124\right)$ y consumo de sustancias $\left(X^{2}=5.83 ; p=.212\right)$ no han sido significativas a nivel estadístico.

Figura 10. Distribución de la percepción de salud mental en hombres, según consumo de tabaco y otras sustancias $(n=300)$

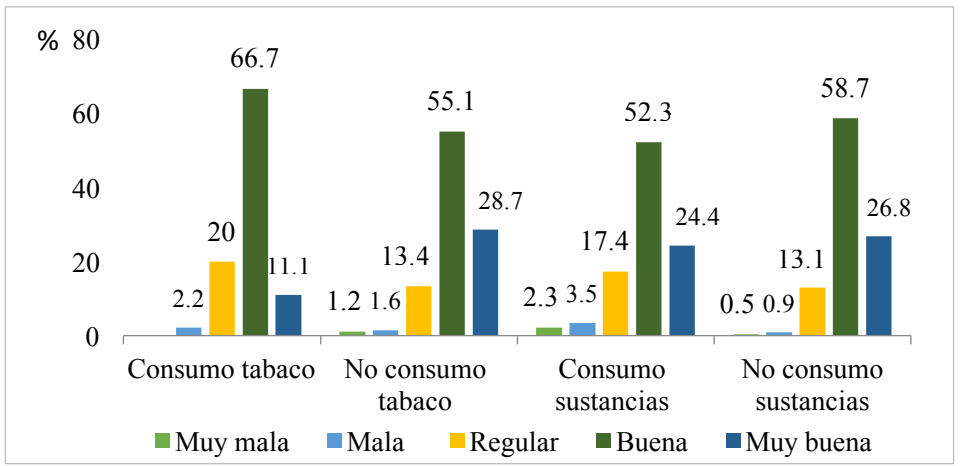

Finalmente, se ha analizado la percepción de salud física en hombres y mujeres según el consumo de tabaco y otras sustancias. El porcentaje de mujeres que valoran su salud física como buena o muy buena es mayor entre las que no consumen tabaco ( $86.7 \%$ vs $83.7 \%$ ), a diferencia de lo que pasa con el consumo de otras sustancias ( $85.6 \%$ vs. $87.4 \%$ ) (ver Figura 11). Pero no se encuentran diferencias significativas, en la percepción de la salud física según el consumo de tabaco $\left(X^{2}=5.19 ; p=.268\right)$ ni del consumo de sustancias $\left(X^{2}=1.85 ; p=.763\right)$. 
Figura 11. Distribución de la percepción de salud física en mujeres, según consumo de tabaco y otras sustancias $(n=300)$

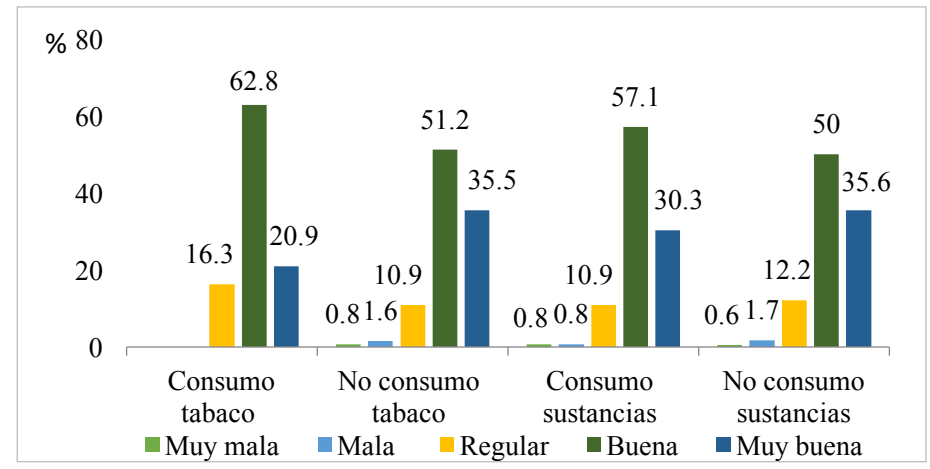

Por último, el porcentaje de hombres que consideran su salud física como buena o muy buena es bastante más alto entre los que consumen tabaco (95.5\% vs $85.4 \%$ ) y algo más alto entre los que consumen otras sustancias (87.3\% vs. 86.8\%) (Ver Figura 12). Tampoco aquí se encuentran diferencias significativas, en la percepción de la salud física según el consumo de tabaco $\left(X^{2}=7.82 ; p=.098\right)$ ni otras sustancias $\left(X^{2}=3.75 ; p=.440\right)$.

Figura 12. Distribución de la percepción de salud mental en hombre, según consumo de tabaco y sustancias $(n=300)$

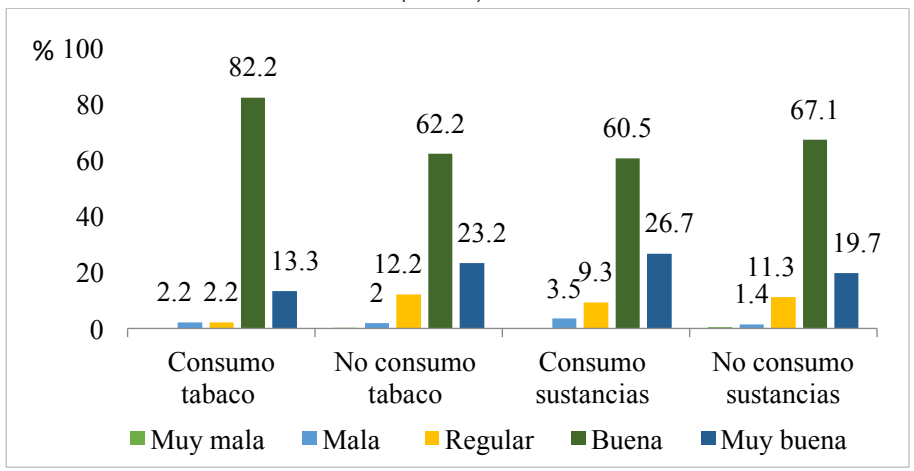

\section{DISCUSIÓN}

A través de este estudio se ha analizado si existen diferencias en el consumo de distintas drogas, según el género, así como el análisis de la percepción de salud mental y física teniendo en cuenta la existencia del consumo, tanto en mujeres como en hombres jóvenes. Este segmento de población, supone uno de los grupos de mayor riesgo (Observatorio Español sobre Drogas, 2019; Plan Nacional sobre Drogas, 2009).

Nuestros hallazgos coinciden con estudios anteriores que muestran cómo hoy en día el consumo de alcohol es el más extendido entre los jóvenes, seguido del consumo de tabaco y otras sustancias (Diatta y Berchtold, 2020; Galán, González y Valencia-Martín, 2014). En concreto, entre las personas participantes, parecen existir diferencias de género en el abuso de alcohol y el consumo de otras sustancias, presentando los hombres una prevalencia mayor en ambas, pero no encontramos diferencias significativas en el consumo de tabaco. Los resultados aquí encontrados son similares a algunos de los hallados por Gómez, Landeros, Noa y Patricio-Martínez 


\section{ANÁLISIS DEL CONSUMO DE SUSTANCIAS E INDICADORES DE SALUD FÍSICA Y PSICOLÓGICA EN HOMBRES Y MUJERES JÓVENES}

(2018), en lo relativo al consumo de alcohol y sustancias, aunque no en lo relativo al consumo de tabaco, ya que estos autores también encontraron que los hombres fumaban más que las mujeres.

El consumo de sustancias en hombres y mujeres jóvenes, especialmente en los hombres quienes han mostrado mayor frecuencia, podría estar asociado a variables como la disponibilidad de las drogas, el inicio temprano, el uso de drogas por parte de los amigos, el bajo riesgo percibido y las actitudes favorables hacia el uso de las drogas (López y Rodríguez-Arias, 2010). Además, otros autores muestran cómo el mayor consumo en hombres se relaciona con otros factores como asistir o no a clase (Medina-Mora et al., 2003).

En nuestro estudio, tanto en hombres como en mujeres, no existen diferencias en la percepción de salud física y mental en función del consumo. Esto podría relacionarse con la baja percepción de riesgo que ha mostrado la población joven (Ruiz, Lucena, Pino y Herruzo, 2010; Suárez-Relinque, Arroyo, Ferrer y Ochoa, 2017) siendo muy poco conscientes de las consecuencias a corto y largo plazo que puede desencadenar el consumo de sustancias, como cambios en los hábitos de sueño y vigilia, pérdida de apetito, cambios en el estado de ánimo, daños pulmonares, cerebrales y otros órganos (Rivera, 2007). Por lo que, una de las hipótesis que podría explicar los resultados del estudio, iría en la línea de aquellos en los que se enfatiza cómo el consumo de sustancias en Ios jóvenes se acompañaría de una baja percepción de riesgo (López y Rodríguez-Arias, 2010; Prada, Bedoya, Cubaque, y Díaz, 2015). En este sentido, algunos autores incluso han mostrado que el consumo moderado y más frecuente de alcohol en hombres y mujeres podría relacionarse con el informe de una mejor salud mental y funcionamiento físico (Green, Perrin y Polen, 2004).

Estos resultados cabría tenerlos en cuenta en base a algunas limitaciones. La primera de ellas podría relacionarse con las características específicas de la muestra que sería interesante ampliar a otros segmentos población en futuros estudios. Por otro lado, el uso del autoinforme podría facilitar la deseabilidad social en las respuestas, si bien estos instrumentos han sido ampliamente utilizados.

Más allá de esto, el estudio enfatiza la importancia de seguir trabajando en la prevención del consumo de alcohol y otras sustancias, dadas las altas prevalencias obtenidas. También la importancia de incluir una perspectiva de género en las estrategias, dadas las diferencias halladas. Más todavía, parece fundamental incrementar el trabajo en los programas de prevención relacionado con la percepción de riesgo y gravedad del consumo porque, según los hallazgos encontrados, los jóvenes no parecen ser conscientes del impacto que estas conductas están generando en su salud física y mental.

\section{REFERENCIAS BIBLIOGRÁFICAS}

Brown, S. A., McGue, M., Maggs, J., Schulenberg, J., Hingson, R., Swartzwelder, S. ... y Winters, K. C. (2008). A developmental perspective on alcohol and youths 16 to 20 years of age. Pediatrics, 121(S 4), S290-S310.

Cruz-Ramírez, V., Gómez-Restrepo, C. y Rincón, C. J. (2018). Salud mental y consumo de sustancias psicoactivas en adolescentes colombianos. Health \& Addictions/Salud y Drogas, 18(1), 97-106.

Diatta, I. D. y Berchtold, A. (2020). Substance use as a function of activity level among young Swiss men. Swiss Medical Weekly, 150(1112), 1-10.

Galán, I., González, M. y Valencia-Martín, J. L. (2014). Patrones de consumo de alcohol en España: un país en transición. Revista Española de Salud Pública, 88(4), 529-540.

Giménez-García, C. y Ballester-Arnal, R. (2017). Cuestionario de Estilo de Vida y Salud para Población Joven. Manuscrito no publicado, Universitat Jaume I de Castellón, España.

Giménez-García, C., Ruiz-Palomino, E., Gil-Llario, M., Ballester-Arnal, R. y Castro-Calvo, J. (2016). Una perspectiva de género en el estudio de conductas de riesgo de los adolescentes. International Journal of Developmental And Educational Psychology: INFAD. 2(1), 189- 198.

Giménez-García, C., Ruiz-Palomino, E., Gil-Juliá, B., Nebot-García, J. E. y Ballester-Arnal, R. (2018). Alcohol y juventud, ¿existen diferencias en consumo de hombres y mujeres según edad de inicio? International Journal of Developmental and Educational Psychology: INFAD. 2(1), 317-328. 
Gómez, Z., Landeros, P., Noa, M. y Patricio, S. (2018). Consumo de alcohol, tabaco y otras drogas en jóvenes universitarios. Revista Salud Pública y Nutrición, 16(4), 1-9.

Green, C. A., Perrin, N. A. y Polen, M. R. (2004). Gender differences in the relationships between multiple measures of alcohol consumption and physical and mental health. Alcoholism: Clinical and Experimental Research, 28(5), 754-764.

Guzmán, R. G. y Ramírez, J. A. (2006). Consumo de alcohol y salud pública. Revista de la Facultad de Medicina de la UNAM. 49(6), 238-241.

Hernando, Á., Oliva, A. y Pertegal, M. Á. (2013). Diferencias de género en los estilos de vida de los adolescentes. Psychosocial Intervention, 22(1), 15-23.

López, S. y Rodríguez-Arias, L. (2010). Factores de riesgo y de protección en el consumo de drogas en adolescentes y diferencias según edad y sexo. Psicothema, 22(4), 568-573.

Lugones, M., Ramírez, M., Pichs, L.A. y Miyar, E. (2006). Las consecuencias del tabaquismo. Revista Cubana de Higiene y Epidemiología, 44(3), [fecha de Consulta 9 de Abril de 2020]. ISSN: 0253-1751.

Medina-Mora, M. E., Cravioto, P., Villatoro, J., Fleiz, C., Galván-Castillo, F. y Tapia-Conyer, R. (2003). Consumo de drogas entre adolescentes: resultados de la Encuesta Nacional de Adicciones, 1998. Salud pública de México, 45(S1), 16-25.

Observatorio Español de Drogas y adicciones. (2019). Estadísticas 2019. Alcohol, tabaco y drogas ilegales en España. Madrid: Ministerio de Sanidad, Consumo y Bienestar social y Secretaría del Estado de Servicios sociales.

Recuperado

de: http://www.pnsd.mscbs.gob.es/profesionales/sistemasInformacion/informesEstadisticas/pdf/20190EDAINFORME.pdf

Östlin, P. (2005). Incorporar la perspectiva de género en la equidad en la salud: un análisis de la investigación y las políticas. Editorial Pan American Health Org. Recuperado de: https://books.google.es/books?id=LZRvaSnhWOC\&pg=PA40\&dq=\%C3\%96stlin, + P. $+(2005) . \& h l=e s \& s a=X \& v e d=0 a h U K E w j t 4 t 0 k 1 N 70 A h V B A m M B H a f$ 3ANMQ6AEINDAB\#v=0nepage\&q=\%C3\%96stlin\%2C\%20P.\%20(2005).\&f=false

Plan Nacional sobre Drogas (2009). Informe de la Encuesta Estatal sobre Uso de Drogas en Estudiantes de Enseñanzas Secundarias (ESTUDES) 2008. Madrid, España: Ministerio de Sanidad y Política Social. Delegación del Gobierno para el Plan Nacional sobre Drogas. Observatorio Español sobre Drogas.

Prada, M. P. P., Bedoya, P. B., Cubaque, M. A. R. y Díaz, G. H. R. (2015). Percepción de riesgo y factores asociados al consumo de drogas legales e ilegales en estudiantes de la Universidad de Boyacá. Enfoques, 1(2), 83102.

Rivera, A. (2007). Factores de riesgo y protección del consumo de drogas entre estudiantes universitarios salvadoreños, año 2007. Revista Crea Ciencia, 4(7), 7-13.

Ruiz, R., Lucena, V., Pino Osuna, M. y Herruzo, J. (2010). Análisis del consumo de drogas legales como el alcohol, el tabaco y los psicofármacos, y la percepción del riesgo en jóvenes universitarios. Psychology, Society, \& Education, 2(1), 21-31.

Salmerón, P., Giménez, C., y Nieto, R. (2016). La promoción de la salud: claves para su práctica. Editorial UOC.

Suárez-Relinque, C., Arroyo, G. D. M., Ferrer, B. M. y Ochoa, G. M. (2017). Baja percepción de riesgo en el consumo de alcohol en adolescentes españoles. Cadernos de Saúde Pública, 33, e00129716

Torres, G. y Fiestas, F. (2012). Efectos de la marihuana en la cognición: una revisión desde la perspectiva neurobiológica. Revista Peruana de Medicina Experimental y Salud Pública, 29(1), 127-134.

U.S. Department of Health and Human Services (2004). The health consequences of smoking: A report of the Surgeon General. Washington, DC, EE. UU.: US Department of Health and Human Services, Centre for Disease Control and Prevention, National Centre for Chronic Disease Prevention and Health promotion, Office on Smoking and Health. 
